\title{
Analytical Research on Upstream Channel Noise Monitoring
}

\author{
Jin Bao Song ${ }^{1, a}$ \\ ${ }^{1}$ Information Engineering School, Communication University of China, Beijing, China
}

\begin{abstract}
The Hybrid Fiber Coax (HFC) network of CATV has tremendous advantages as the platform of B-ISDN. In practical application, the upstream channel noise and the special tree-and-branch topology will make it difficult to transmit data. Hence it's necessary to monitor and analyse the real-time characteristics of the upstream channel noise and bit-error-rate (BER), and understand present and historical situation of noise. In the paper, the cause and characteristics of each kind of noise especially the noise in the upstream channel are studied. Based on this, the formula to calculate the BER of QPSK and 16QAM signal in various environment of upstream channel noise is developed respectively. The imitation of system is made. And the performance comparison between them is given.
\end{abstract}

\section{Introduction}

The broadband network based on two-way HFC cable network has the advantages of wide users, low cost, wide band and good compatibility and therefore has a broad development prospects. But there's a lot of noise in upstream channel of HFC network, combined with the funnel effect of the noise of coax cable distribution network, which seriously influences the upstream transmission of digital signal and limits the development of two-way HFC network[1]. So effective measures should be taken to suppress the noise in return path. To suppress noise, the characteristics of the noise must be firstly deep understood. The noise of actual network must be tested and analysed. So that the method of being targeted to suppress noise will be generated. This paper is based on this consideration. A set of real-time monitoring test system of cable TV upstream channel noise is designed and set up. And the test data is analysed scientifically[2]. The noise characteristics are extracted. The historical information of noise is stored. The digital transmission characteristics and noise performance of upstream channel as well as their change trend can be timely understood by the user. Thus reasonable suggestions to two-way network maintenance and upstream business can be put forward[3].

HFC upstream channel monitoring systems that can be seen on the market at present are basically the company's products, such as TRILITHIC Company's GUARDIAN / 9580 reverse maintenance system, ACTERNA PathTrak performance monitoring system and so on. These systems generally have the function of real-time monitoring upstream channel noise spectrum and adopt their own software to analyse noise spectrum in order to achieve the alarm function[4]. Also a lot of long-term historical data are stored for the researchers.

\footnotetext{
${ }^{\mathrm{a}}$ Corresponding author: songjinbao@cuc.edu.cn
}

But the existing monitoring systems only simply collect and record the noise data for simple alarm. They don't give the user an intuitive, simple and clear illustration about upstream channel. Only very professional and technical personnel can understand the channel condition through analysing these data. This is obviously not conducive for the normal network management personnel to do the upstream channel monitoring and timely maintenance. In order to solve this problem, we hope to develop the noise test system of cable back analysis software in this paper, which realizes the comprehensive analysis of the test data. And the results of the analysis feedback to the user in the most practical way. The nonprofessional users can also understand the general operation of the network and other useful information of network maintenance in the present condition[5].

\section{The integral design of the monitoring system}

The main purpose for monitoring system is to understand the noise characteristics of HFC network upstream channel. The real-time situation and historical situation of the noise are analysed. The real-time monitoring of the whole upstream system is realized. The corresponding reports and recommendations are put forward.

In order to measure the noise of the transmission network, the generally adopted method is to send a test signal, pass the network transmission and receive the signal. By comparing the difference between the signals before and after the network transmission, the network noise's influence on the signal is analysed. In HFC network, this method means that a digital signal for test is launched by the user, passes the upstream channel of HFC network transmission, the signal is received on the 
front end. By comparing with the known test signal, it is concluded that the upstream channel noise effect on the digital signal transmission. This is the method used by all current HFC upstream channel monitoring system. But this test method has the certain shortcomings. First of all, the cost of the whole test system would be high if the client launch test signal and compare on the front end. Secondly, the operability of the test system will be greatly reduced. In order to overcome the above shortcomings, considering the accuracy, operability and the overall cost of the test system, an improved testing method is adopted in this system. All of the measuring instruments will be put in the front end, through the corresponding processing, to simulate the digital signal transmission in HFC network upstream channel.

Based on the above consideration, the test system is designed as shown in Figure 1. The whole test system is divided into the following five parts: the noise import part, the spectrum test part, reverse modem part, the error test part and test result analysis part.

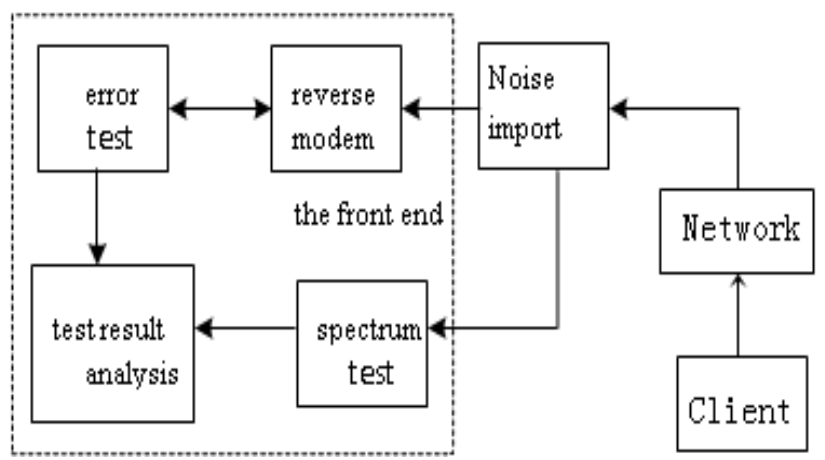

Figure 1. The integral design of the monitoring system

The noise import part is introduced to analyse the noise function. In this scheme, all of the test equipments including signal source equipment are on the front end. So the test signal does not actually pass through the upstream channel transmission of HFC network. In order to make the test result manifests the influence on transmission signal of the upstream channel noise, noise interference to signal transmission in HFC network must be imitated by adopting corresponding way. We usually think that HFC network upstream channel noise belongs to the additive noise. So signal and noise can be separated. On the one hand, the signal modulation in the client is implemented on the front end. On the other hand, the noise of the client is introduced into the front end. The demodulation test is made after adding noise to the modulation signal through the coupler. In this way, the test system can imitate signal transmission in actual HFC network. The role of the noise import part is to introduce the upstream channel noise into our test system.

The function of the spectrum test part is to use a dedicated cable analyser to monitor the frequency spectrum characteristic of the upstream channel noise in non-business state. The noise signal can be introduced by the noise import part. Because we need to know the upstream channel frequency range $(5 \sim 65 \mathrm{MHZ})$ noise distribution. Therefore, parameter setting and measurement of cable TV analyser is different from the downstream channel. The test result of cable analyser to is transmitted to the computer through GPIB cable. The corresponding analysis and the test data processing are carried out by the computer.

The function of reverse modem part is modulation and demodulation to digital signal in some way. Because the digital signal from the source is a baseband signal, which can't transfer in the upstream channel of cable TV system. So the signal modulation and demodulation must be firstly made when error characteristics of upstream signals is measured. And the modem method is different from the process of signal transmission in downstream channel. So it is called reverse modulation and demodulation.

The error test part is to do the digital signal bit error rate test by using a dedicated error analyser. The error analyser is put as signal source to send baseband digital signal. Baseband digital signal passes through reverse modulator, superimpose upstream channel noise introduced from the former part, passes through the reverse demodulator and arrives at the receiving end of the analyser. So that the error characteristics of upstream channel can be concluded. Error test result of error analyser is transmitted to computer via RS232 cable. Further analysis of the data is carried out by computer.

Test result analysis part belongs to the software. The computer statistically analyses error and noise characteristics and obtains the corresponding analysis results according to the test data transmitted from the error analyser and cable TV analyser. If performance deterioration of the upstream channel happens, the system will generate real-time alarm information, put forward corresponding suggestions and notify the manager timely troubleshooting. At the same time, the analysis software also will store the historical information of the system, analyse the long-term trend the upstream channel performance and put forward practical suggestions for the network maintenance and business configuration problems.

\section{The implement of the monitoring system}

The schematic diagram of HFC upstream channel noise monitoring system is shown in Figure 2.

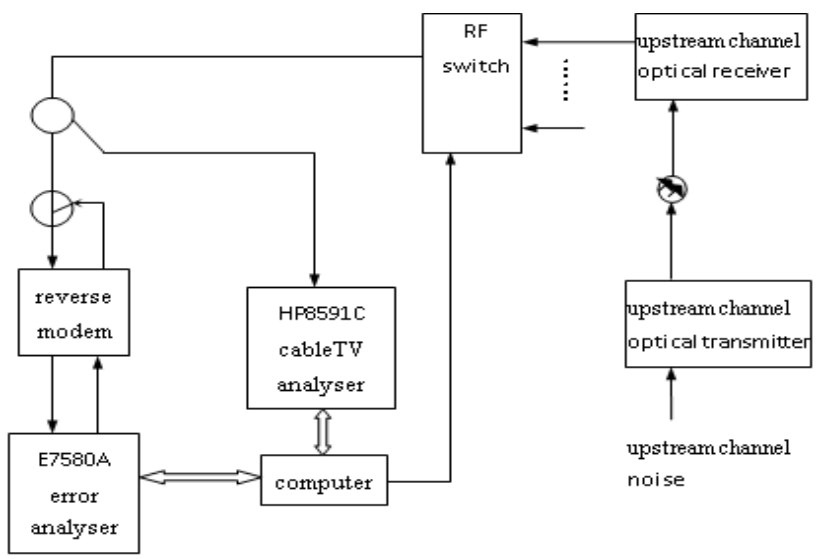

Figure 2. The schematic diagram of HFC upstream channel noise monitoring system 
In this paper, the monitoring system are simulated by using the MATLAB software. The way is as follows:

(1)A random testing signal is sent.

(2)The random testing signal passes through QPSK and 16 QAM modulation respectively in two ways.

(3) The random testing signal passes through the network.

(4) Gaussian noise, narrowband continuous wave noise and impulse noise signals are introduced respectively.

(5) QPSK and 16 QAM demodulation are accordingly made.

(6) Bit error rate and other indicators are given by the monitoring system.

(7) The noise's influence on the signal in the network is analysed by comparing the difference between the signals before and after the transfer.

In HFC network, this method means that a digital signal for test is launched by the user, passes the upstream channel of HFC network transmission, the signal is received on the front end. By comparing with the known test signal, it is concluded that the upstream channel noise effect on the digital signal transmission. By comparing differences of various indicators of different types of noise in different signal under different modulation mode, the best way of modulation and demodulation adopted to different upstream channel noise are determined. This test method through MATLAB simulation is the current method of all HFC upstream channel monitoring systems use. The various cable TV systems upstream channel test data and indicators of this test system are required for every cable channel statistics. In addition, the research on the upstream channel cable TV system performance in this paper is somewhat superficial, but also carries on the beneficial attempt for the future development and reconstruction of the cable television.

\section{The simulation of HFC network upstream channel}

In HFC network, general transmission general transmission the upstream channel is the digital signal. Therefore, BER should be chosen as main indicators to evaluate its transmission performance. But in fact, the analog signal is in the process of transmission. All kinds of noise has a direct effect on the analog signal, which in turn affect the analog signal correctly received on the front end. Therefore, the requirement of digital signal transmission error rate can be attributed to the requirement of the analog signal carrier to noise ratio on the receiving end. In the test system, the carrier to noise ratio largely reflects the channel noise, with the characteristics of real time measurement. The error rate measured by the error analyser has only a posteriori. So we need to further study the relationship between the carrier to noise ratio and the error rate and analyse channel noise influence on BER. Due to the different modulation mode, the relationship between BER and carrier to noise ratio is different. So the main content of this paper is to discuss the most commonly used two kinds of modulation: QPSK and 16 QAM mode in HFC network upstream transmission mode and all kinds of noise influence on signal bit error rate. The corresponding simulation is carried out by using MATLAB software.

\subsection{Upstream channel BER of QPSK modulation}

In QPSK modulation, baseband signal waveform, the waveform and spectrum of modulated signal, as well as the signal constellation diagram are shown in the case of not setting the output parameters, as shown in Figure 3.
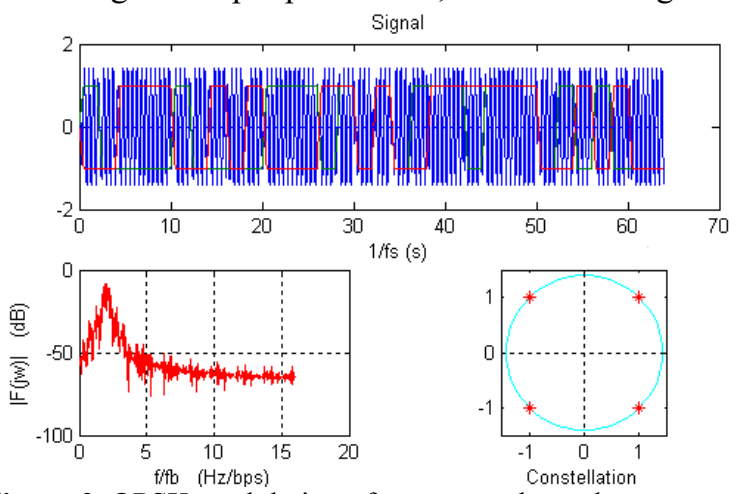

Figure 3. QPSK modulation of upstream channel
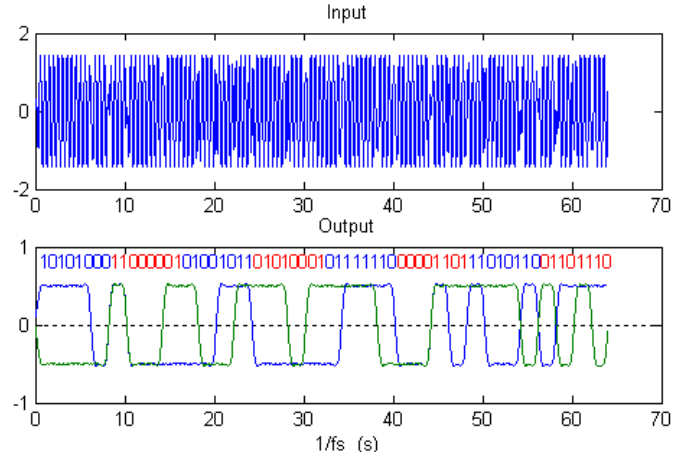

Figure 4. QPSK demodulation of upstream channel

Based on the analysis of HFC network upstream channel noise effects, noise actually always is introduced into the main categories: Gaussian noise, narrowband continuous wave and impulse noise. The following analysis is according to the characteristics of these three kinds of noise.

(1) Gaussian noise

After Gaussian noise superimposed on the transmission signal in upstream channel passes by receiving filter, it becomes a narrowband Gaussian noise, which can be represented as:

$$
n(t)=n_{i}(t) \cos \omega_{c} t-n_{q} \sin \omega_{c} t
$$



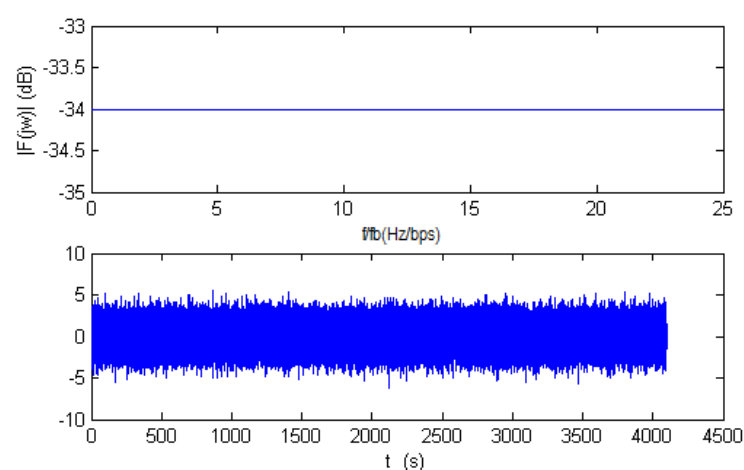

Figure 5. Gaussian noise and spectrum in upstream channel

(2) Narrowband continuous wave noise

After continuous wave noise superimposed on the transmission signal in upstream channel passes by receiving filter, it becomes a narrowband continuous wave noise, which can be represented as:

$$
c(t)=a \cos \left(\omega_{c} t+\Delta \omega t+\theta\right)
$$

Narrowband continuous wave noise and its spectrum are shown in Figure 6.

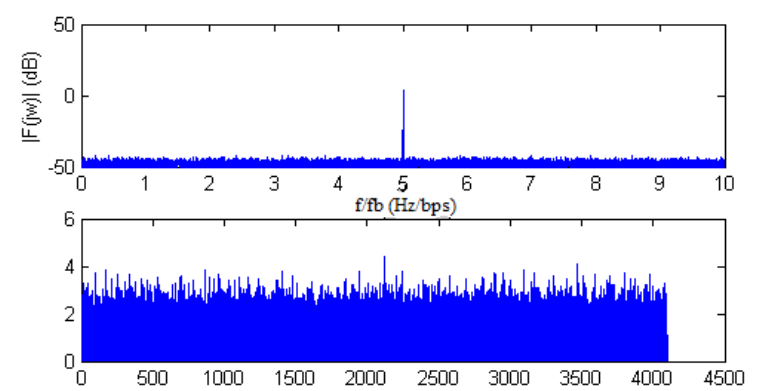

Figure 6. Narrowband continuous wave noise and spectrum in upstream channel

\section{(3) Impulse noise}

Hypothesis is as follows: Impulse noise model is the Poisson impact sequence. Random arrival. Each impulse area is independent and obeys the Rayleigh distribution. The sample is expressed as:

$$
i(t)=\sum_{i=0}^{\infty} a_{i} \delta\left(t-t_{i}\right)
$$

7.

Impulse noise and its spectrum are shown in Figure
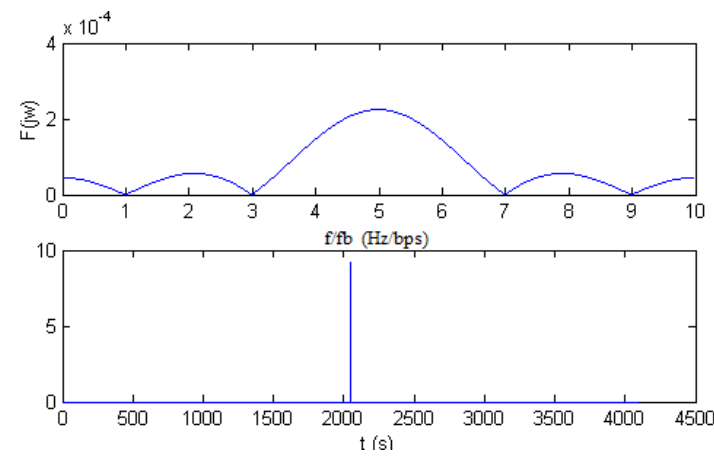

Figure 7. Impulse noise and spectrum in upstream channel
Random binary signal generated by a random binary signal generator and its spectrum are shown in Figure 8.
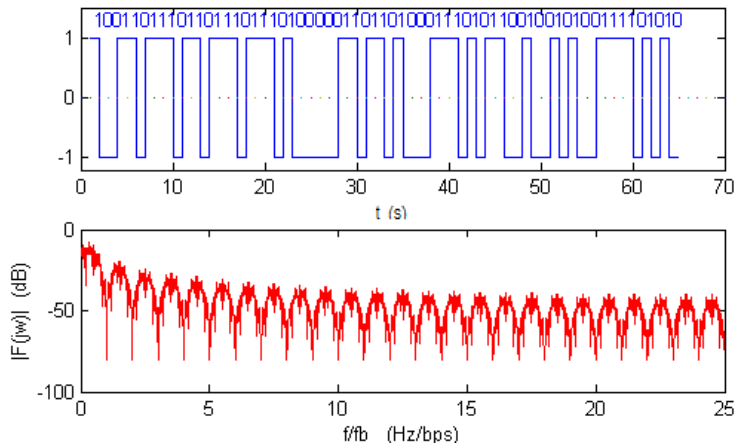

Figure 8. Random binary signal and spectrum in upstream channel

The simulation waveform, spectrum and the constellation diagram of the random binary signal after QPSK modulation are shown in Figure 9.
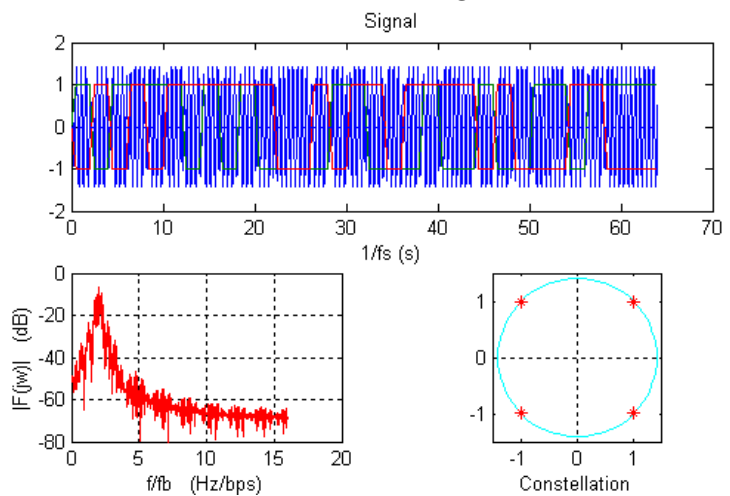

Figure 9. Random binary signal after QPSK modulation in upstream channel

Gaussian noise shown in Figure 5 is introduced into upstream channel.

The waveform after QPSK demodulation is shown in Figure 10.
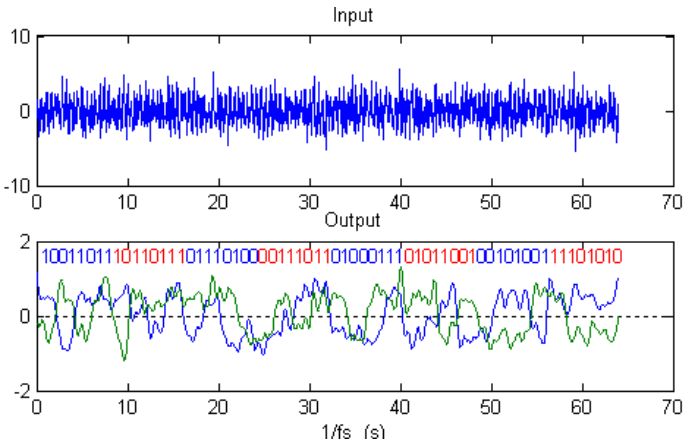

Figure 10. The waveform after QPSK demodulation in upstream channel

The comparison between the original input signal and the final output signal is shown in Figure 11. 

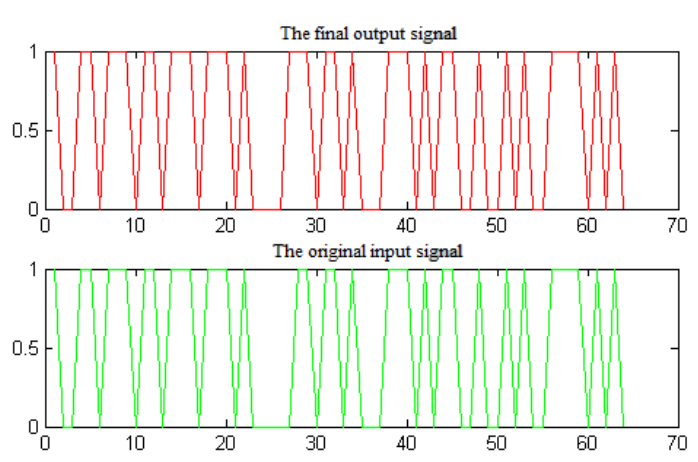

Figure 11. The comparison between the original input signal and the final output signal in upstream channel

\subsection{Upstream channel BER of QAM modulation}

In 16QAM modulation, baseband signal waveform, the waveform and spectrum of modulated signal, as well as the signal constellation diagram are shown in the case of not setting the output parameters, as shown in Figure 12.
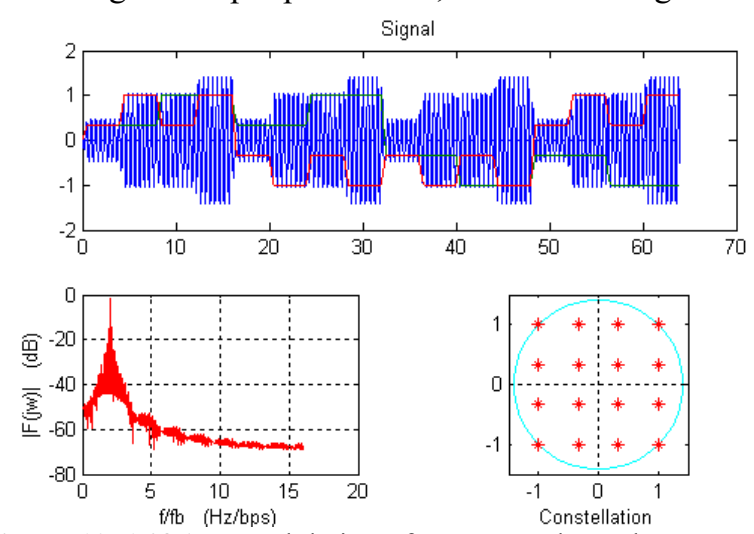

Figure 12. 16QAM modulation of upstream channel
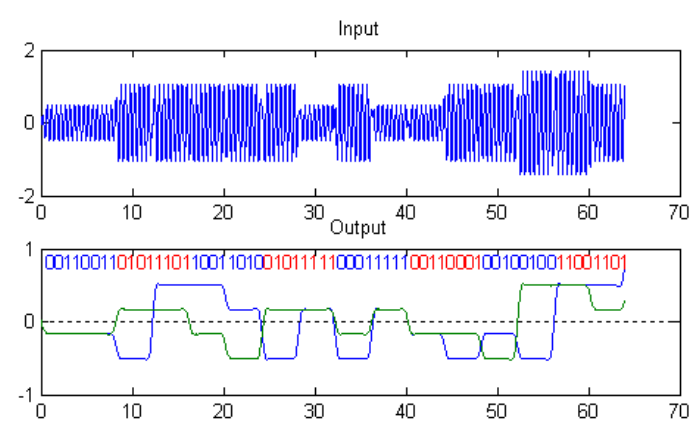

Figure 13. 16QAM demodulation of upstream channel

Random binary signal generated by a random binary signal generator and its spectrum are shown in Figure 8. The simulation waveform, spectrum and the constellation diagram of the random binary signal after 16QAM modulation are shown in Figure 14.
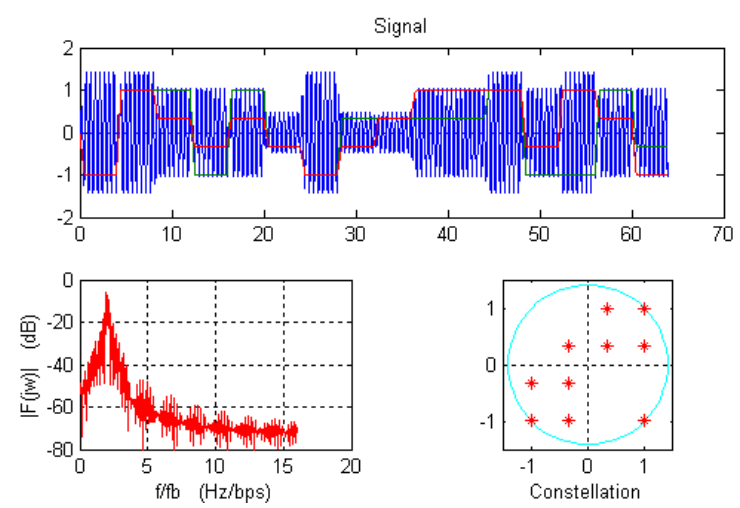

Figure 14. Random binary signal after 16QAM modulation in upstream channel

Gaussian noise shown in Figure 5 is introduced into upstream channel. The waveform after 16QAM demodulation is shown in Figure 15.

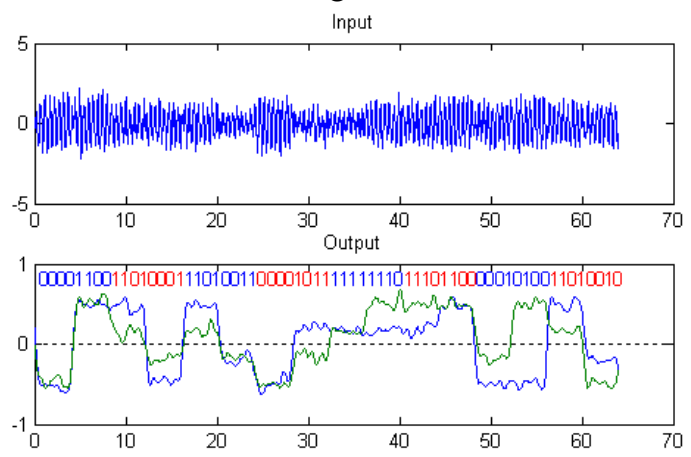

Figure 15. The waveform after 16QAM demodulation in upstream channel

\section{Summary}

In practical application, the upstream channel noise and the special tree-and-branch topology will make it difficult to transmit data. Hence it's necessary to monitor and analyse the real-time characteristics of the upstream channel noise and BER. In the paper, the cause and characteristics of each kind of noise especially the noise in the upstream channel are studied. The research and application of this topic have wide development prospects.

\section{References}

1. Paul T S. Two-Way Cable Television System Characterization. SPIE Proc, Vol 2609, Philadelphia, Oct, 2009, 94-104.

2. Eldering C A. CATV Return Path Characterization for Reliable Communications. IEEE Commun Mag, 2010, 33(8):62-69.

3. Wolters R P C. Characteristics of Upstream Channel Noise in CATV-network. IEEE Trans. On Broadcast, 2011, 42(4):328-332.

4. Kapoor S. Mitigating Impulse Noise over HFC Network For Telephony-Over-Cable. IEEE GLOBECOM, SESSION 28, 2012, 967-971.

5. Campos L A, Krinsky D. Effects of Upstream Channel Distortion in HFC Networks.SPIE Proc, Vol 2609, Philadelphia, Oct,2009:203-214. 